\title{
Research on Environmental Ecology in Contemporary Landscape Design
}

\author{
Li Wang \\ School of Art \\ Hubei University of Education \\ Wuhan, China
}

\begin{abstract}
Environmental ecology is an important idea in landscape design in recent years well received by audiences because it combines ecology with humanity and service of landscape. This paper designs environmental landscape with environmental ecology, follows the original ecological foundation of project and comprehensively plans spatial arrangement of landscape area and introduces design methods of road, vegetation and featured landscape in detail.
\end{abstract}

Keywords - environmental ecology; landscape design; service; humanity

\section{INTRODUCTION}

In today's society, with the continuous acceleration of industrialization and urbanization, natural resources are consumed and destroyed seriously and the ecological systems also degrade seriously, causing environmental problems such as desertification of land, water resource shortage, reduction of forest resources, and absence of wetland biodiversity and acceleration of species extinction which have restricted the sustainable and stable development of human society.

China brings ecological civilization construction in overall layout of socialist construction, proposes the policy of economization first, protection first and spontaneous recovery first and begins to construct beautiful China. As the discipline that coordinates the relationship between human and nature through planning and design, landscape plays an important role in China's ecological civilization construction. Sassakii, the deceased leading authority in landscape design, said, landscape designers can bring profound changes for the earth. Similarly, he may be fascinated by self-expression in trivial arts. With the responsibility for society and land, landscape designers will choose the former. The emergence of environmental ecology provides a new guiding thought for today's landscape designers and makes the discipline of landscape design have a historical responsibility.

\section{CONCEPTS AND THEORIES}

\section{A. Environmental Ecology}

At present, the comprehension of ecology presents different characteristics in different regions. Generally speaking, ecology can be a comprehensive environment including human and nature and the adaptation of species to environment. The concept of ecology is proposed by Haeckel (1866) who thinks ecology refers to a discipline related to the relationship between organism and the surroundings. The research object of ecology develops from animals and plants to human and the research contents incline to the relationship between human and environment.

The relationship between human and environment forms the ecological system which refers to the indispensable system formed between biomes through mutual interchange of material, energy and information in environment within a certain period of time and certain space. In the traditional social development, both typical agricultural civilization in China and industrial civilization acquire materials in natural environment to promote production development and discharge wastes into natural environment, leading to ecological crisis like shortage of natural resources, desertification of the grassland and environmental pollution. The increasingly bad ecological environment leads to species extinction and makes living environment of human degrade continuously. People realize the significance of protecting natural environment in the development. We can reduce and get rid of the pollution of wastes and maximize economic benefits through changing the way of resource utilization and transforming resources into products to utilize circularly.

Environmental ecology is a new civilization form formed under this condition to effectively solve problems in the development of agriculture civilization and industrial civilization and realize harmony of human and nature.

Environmental ecology has an important position in modern landscape design. At the end of the 19th century, some designers with sense of social responsibility in western countries realized the thought of ecological protection should embody in landscape design and integrated ecological ecology in landscape design.

Modern environmental ecology comes from western industrial countries and refers to one of the most influential ideological trends disproving Western Classicism and the superiority of mankind. The landscape design of environmental ecology still insists the thought of people first but it has longterm consideration of it. It considers the attentions paid to human, thinks the influences of human behaviors on the environment and sublimates into the thought of nature oriented environmental ecology. Different from the people-oriented 
thought, the nature oriented thought of environmental ecology emphasizes interdependence between human and nature, pays attention to the diversity of nature and advocates the design methods of virtuous cycle. It advocates the principle of joint prosperity in design to avoid human to become the ecological refugee in nature.

\section{B. Introduction to Environmental Landscape}

1) Meaning of environmental landscape: Landscape first appears in Septuagint to describe the scenery of Jerusalem. Therefore, landscape is first used to describe scenes of city. Afterwards, the description object of landscape expands from city to village. It expresses sceneries and people's experience in enjoying the sceneries. According to the natural explanation, landscape mainly refers to natural process and the trace of human activities. As an aesthetic object, landscape expresses people's attitudes toward nature, human and land as well as human and city, reflects human's ideal and desire; as a place of human life, landscape people's orientation in space and place identification; as a symbol, it is the trace of activities of human and nature and the interaction of them. Therefore, landscape has properties of aesthetic appreciation, experience, science and trace.

2) Types of environmental landscape: According to functions, environmental landscape is divided into park landscape and residential and commercial landscape. Park landscape takes leisure area as the main part, including natural landscape and artificial landscape. Natural landscape includes terrain, landform, water source and vegetation; artificial landscape includes sound and light. The comprehensive residential and commercial landscape expresses the commercial and residential functions, such as the high-rise residential and commercial complex of Wanda.

According to geographical location of landscape, it is divided into mountain landscape and water landscape. Mountain landscape refers to the landscape area built beside mountains and makes the best of landform and vegetation and embodies the deep communication between human and natural environment. Water landscape includes landscape beside river, sea, lake and continent as well as island built beside water, forming comprehensive landscape of landscape area and water environment.

According to landscape structure, it consists of natural landscape and human landscape. Natural landscape includes landscape built beside mountain and water; human landscape focuses on sculpture, sound, light and pavilions, terraces and open halls in urban area.

Environmental landscape also includes urban and rural landscapes.

\section{Meaning and Features of Landscape Design}

Landscape design means using technical methods to create aesthetic places through terrain transformation, plantation of flowers and trees and construction of buildings for people to appreciate and have a rest. Landscape design belongs to a comprehensive specialty with extensive range in architecture.
With the development of human society and city, landscape design has gone through complicated presentation process. Connotations, methods and development spaces of landscape design continuously express colorful and eyeable contents and forms and embody the dialectical development of inheriting in development and developing in inheritance.

Landscape design should be people-oriented to consider users' habits and belief and meet the demands of them. It can be adjusted according to users' demands after the design. Besides, in landscape design, we should fully consider the cost and landscape benefits which are foundations for smooth completion of landscape. Under the influence of economic features, at present, many landscape designers use low price materials easy for maintenance, surrounding facilities and local materials to reduce transportation expense. Lastly, as the main participator in landscape design, designers have absolute speaking right. The different knowledge capabilities and ways of thinking of designers make ever-changing landscapes.

\section{APPLICATION OF ENVIRONMENTAL LANDSCAPE DESIGN BASED ON ENVIRONMENT AL ECOLOGY}

\section{A. Design the Landscape Area}

In this design, the project depends on Big Nanshan in the west, overlooks Shenzhen Bay in the east and is next to the developing Sea World financial district with superior geographical environment but the traffic is inconvenient "Fig. $1 "$.

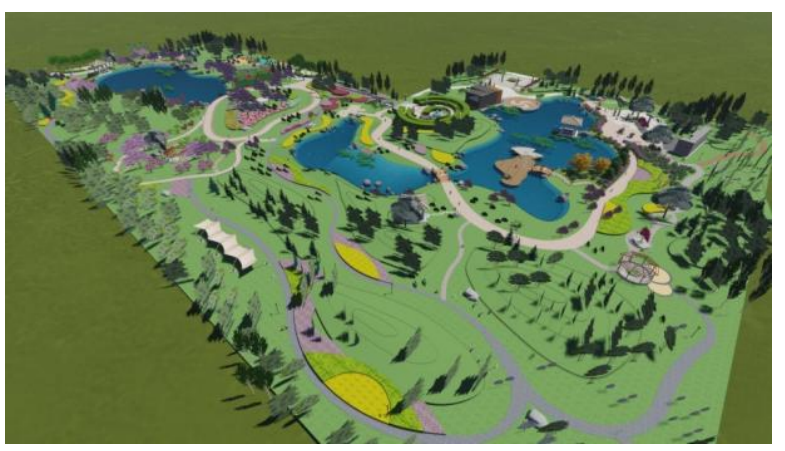

Fig. 1. Comprehensive landscape map.

\section{B. Space Plan and Design}

Reasonable landscape design should first meet function needs according to the existing natural conditions. In this project, because the land for project use is deserted flat ground without the influence of mountain and river, the terrain factor has little influence on landscape design. In design, it is necessary to take advantage of terrain and make the best of water environment. As shown in "Fig. 2", the overall space of landscape includes two artificial lakes. Roads and vegetation surround the lakes to increase the ecology of landscape. At the meantime, footpaths in the landscape connect different areas to meet the requirements of traffic. 


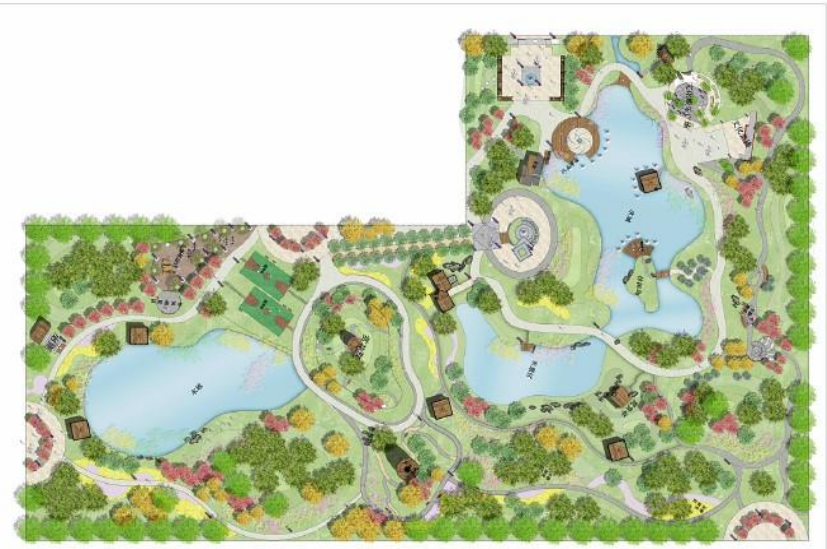

Fig. 2. Project planning map.

Established in the center of landscape, the fountain is surrounded by green trees to increase the safety of landscape. The sense of depth is ensured in design. After the fountain opens, it forms contrast of height with dwarf trees around and the lakes in the distance "Fig. 3".

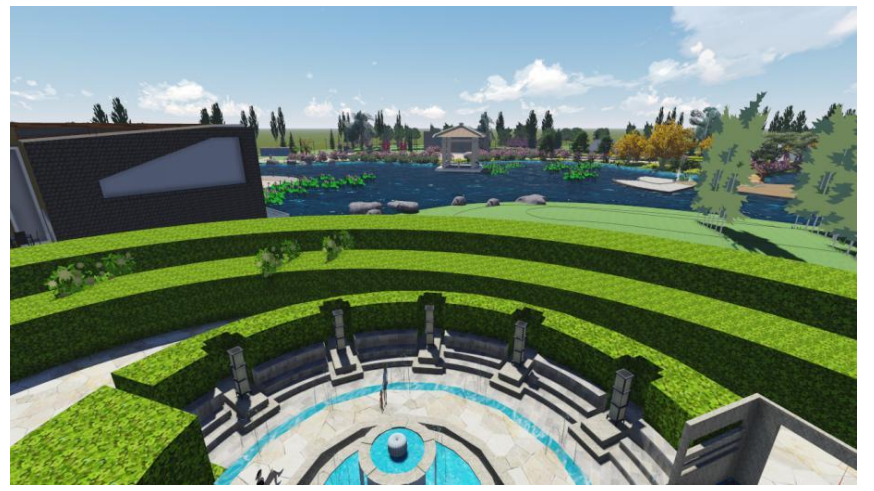

Fig. 3. Center district planning.

Lush vegetation landscape and broad lake landscape appear after design. In order to conform to terrain and make the most of natural landscape in landscape design, the buildings adopt backward terrace and make consumers furthest appreciate the beautiful natural landscape.

The combination of terrain with landscape design forms strong sense of closure. The ecological, personalized and diversified inner space is designed according to landscape layout and regional characteristics. Natural landscape with growth of mangrove and inhabitation of birds reappears on the artificial lake in the project to realize extension and unification of space. The landscape design adopts clear soft landscape design, divides trees, shrubs and grassland into different space levels and chooses trees according to local climate conditions. The integration of local traditional elements and modernized design methods of foreign countries makes the landscape in the garden diversified and colorful. The "people-oriented" landscape design creates the landscape space to meet different requirements.

\section{Landscape road embodies artistic charm}

Road landscape design integrates contact space with living space. The dendritic or cyclic end break roads extend to the interior of landscape in order to make the road can reach each scenic spot. The layout of road should be vivid and flexible. In this project, the landscape layout and road planning carry out according to characteristics of project. The roads make a distinction between the important and the lesser one and have diversified forms according to lakes to make the roads in the landscape flexible and winding but have good accessibility. The landscape layout and road extension coordinate mutually and integrate closely. Like the framework of landscape, roads organically connect buildings with scenic spots to embody integrality "Fig. 4".

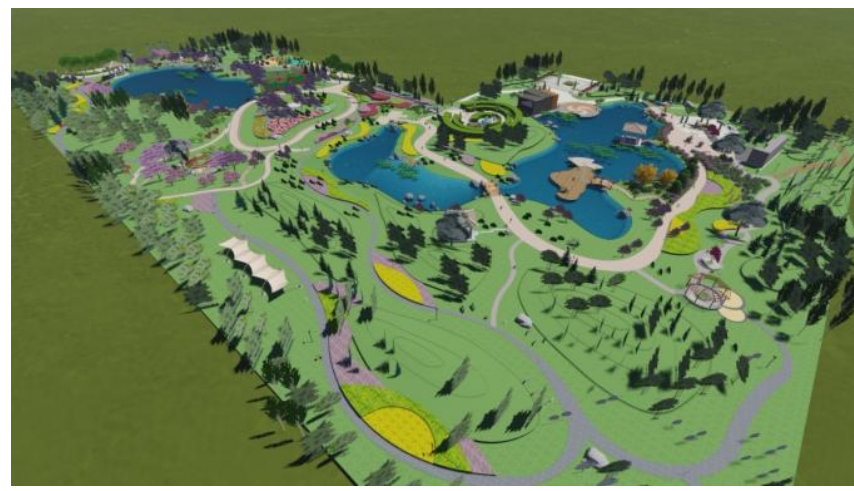

Fig. 4. Road design in landscape area

Ground pavement in landscape design adopts multiple techniques to create different visual experience. Winding roads make people feel the winding path leading to a secluded spot. It often uses streamline pavement. Wide road makes people feel open so we large and rich pavement materials can be used to reduce this feeling; footpaths make people feel narrow so pavement materials with small area can be used to express its delicacy.

Rich techniques can be used in ground pavement because of the diversified road forms. For example, the wide roads in the center of the landscape use traditional simple pavement, while the landscape footpaths use wood and pebbles. The waterborne platforms use marble pavement to create a simple and natural atmosphere "Fig. 5".

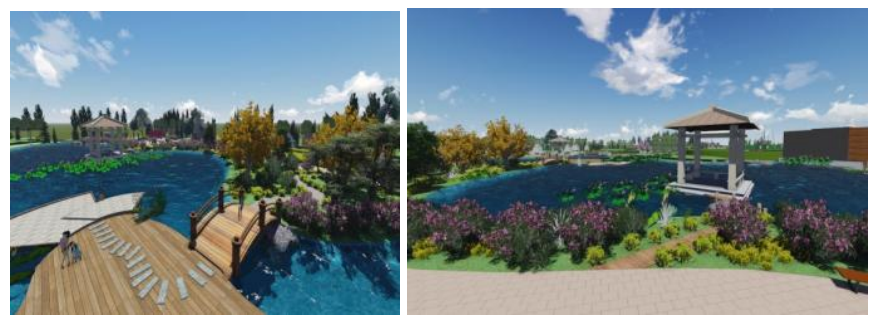

Fig. 5. Landscape road pavement figure

\section{Plant Landscape Design}

Collocation of plants is distinctly important in landscape design. It should follow the following principles: seasonal change, color matching of buildings, random strewing of plants, 
collocation of varieties, collocation with featured landscape, in order to create rich natural landscape.

The landscape design in this project makes the best of the original natural landscape, analyzes the style of buildings and adopts various kinds of plants "Fig. 6".

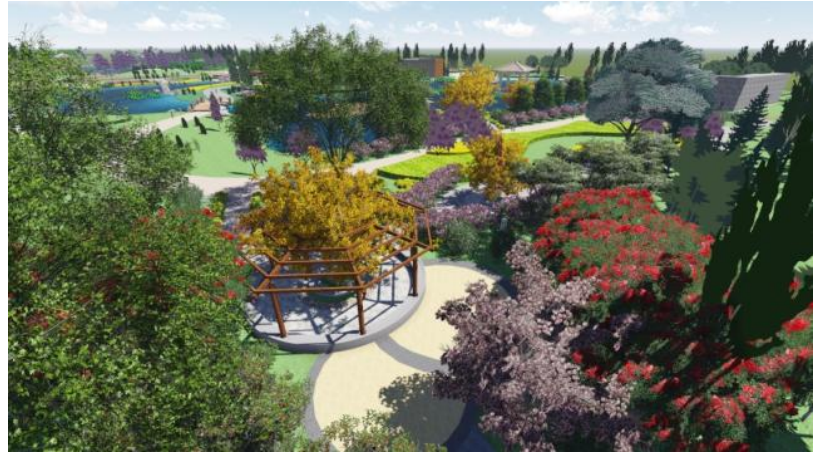

Fig. 6. Landscape

For example, some greening center uses different kinds of plants such as shrubs, trees, broad and narrow leaved plants. The plantation of plants skillfully matches with benches and water system in the landscape area. The rich gradation and various forms make the landscape have distinct local features. The landscape layout fully considers its function and uses different plants to express different themes, which have strong ornamental value.

\section{E. Featured Landscape Design}

The featured landscapes have various kinds. Waterborne platform and stepping stone on water surface are designed beside water system; tables and benches are set among lush trees, in order to make the landscape peculiar with strong ornamental value and practicality. As shown in "Fig. 7".

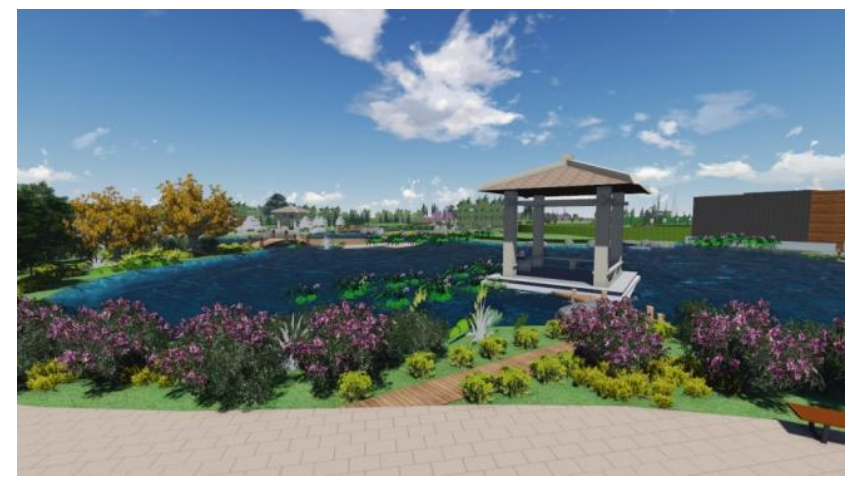

Fig. 7. Featured landscape design

\section{DESIGN PRINCIPLES AND METHODS}

\section{A. Design Principles of Environmental Landscape in This Project}

Although environmental landscape has different divisions, it should meet leisure and environmental requirements. The design principles are as follows:
First, the service principle of landscape. Environmental landscape is the integration of environment. Both natural landscape and artificial landscape are to meet people's demands of relaxation. In order to provide opportunities for users to get close to nature and relax mind and body, the environmental landscape should be serviceable.

Second, the principle with integration of naturalness and humanity. Environmental landscape includes natural landscape and human landscape. In landscape area, the two landscapes embody harmony and unity to promote landscape construction. For example, the combination of natural vegetation and road meets the demands of traffic and greening; the combination of landscape with lighting design creates aesthetic artistic conception.

Third, the principle of uniqueness and pertinence of environmental landscape. Landscape area has different terrains and landscapes and climate differences. Under the influence of climate and terrain, environmental landscape shows regional particularity and pertinence of different landscape area. The historical and cultural differences also form particular environmental landscape.

Fourth, principle of change of landscape. Landscapes are first designed by environmental designers and embody their personal characteristics. After used by consumers, because of the serviceability of landscape, if it fails to meet users' demands, even the most distinct landscape will be abandoned. Therefore, environmental landscape should be changeable especially in public facilities and vegetation. In some landscape areas, the square is changed into parking lot and basement parking is built, or the area and kind of vegetation changes.

\section{B. Application Methods of Environmental Ecology in Environmental Landscape Design}

1) Combination of environmental landscape design with environmental ecology: The environmental landscape design should fully reflect the idea of environmental ecology, which means we should realize landscape embodies the harmony of human and nature. Therefore, we should consider the naturalness of environmental landscape, pay attention to characteristics of natural resources and vegetation, combine natural resources with social resources and adhere to the idea of environmental ecology. The requirements of environmental protection and sustainable development should be met in overall layout, utilization of materials and later maintenance in the design.

2) Integration of landscape with environmental ecology in traditional Chinese landscape art: China has a long history of more than five thousand years and continuous cultural tradition. Therefore, Chinese people have special feelings for traditional culture. Meanwhile, traditional Chinese culture has huge and profound effects on social development. China has exquisite art beyond comparison. The great charm and extensiveness of traditional Chinese culture and art influences our modern life and has great influence on modern landscape construction. 
China is a country with an ancient civilization and a history of five thousand years. All of us are edified by traditional culture, in which people can feel the most simple and warmest power. In landscape construction, the integration of emotions in design and skillful application of traditional Chinese culture and art can make audiences find emotional resonance and attract their attentions to realize the best landscape effect.

3) Attentions paid to integrity and particularity of landscape design: The number of landscape design in our country increases gradually. It is important to carry out landscape design of environmental ecology through organic integration of landscape space with functions, layout and style of buildings, terrain and road. Landscape designers first implement ecological design, furthest reserve the original natural landscape and regional characteristics; design roads according to their using functions instead of deliberately creating winding forms to pursue forms that influence the usage; scientifically and reasonably plant plants. For example, two types of plants should be planted on both sides of the main trunk road in the landscape area. High trees can obstruct noise of motor vehicles and shrubs can form green belts to divide space and protect pedestrians. Plants without thorns that will not cause allergy should be provided.

4) Landscape function pursues the coordination of naturalness and practicality:Landscape designers should achieve aesthetic property and practicality as well as functionality. However, at present, the environmental landscape design has problems such as single function of landscape space and unreasonable function design. For example, the environmental landscape design is single space for appreciation, leisure or activities instead of integrating these functions. It relates to land shortage and design in landscape area. Although the available space area in park is small, the diversified landscape functions can be realized through reasonable, effective and uttermost utilization of landscape space.

Therefore, we should realize diversified landscape functions according to physical truth in environmental landscape design. For example, create waterscape to purify air and beautify the environment. Moreover, construct places for activities and some steps beside waterscape, or design some sunken event squares to serve temporary gathering or activities, in order to make the functions of landscape design more diversified and strengthen its sustainable utilization.

\section{CONCLUSION}

This paper fully considers and makes the most of geographical environment and natural landscape of bases to make the landscape design have strong geographical characteristics and identification. The ecological design of landscape is comprehensive and embodies people orientation. Afforested square is built according to different landscape nodes and geographical environment, so that people can have good visual experience and beautiful psychological feelings and appreciate landscape in the garden.

\section{REFERENCES}

[1] Chen Lu. Research on Garden Environment in Urban Community [M], Beijing: China Forestry Publishing House, 2007: 123

[2] Shen Qingji, Shi Yan. Thinking on Social-ecological Relationships in Ecological Residential Community [J], Urban Planning Forum, 2013(3): 45-48

[3] Li Zhihong. Discussion on Control Elements of Landscape Design in Residential Quarter [J], Guangdong Architecture Civil Engineering, 2015(7): 33-45

[4] Wang Manman. Discussion on Understanding of Ecological Compensation Design [J], Shanxi Architecture, 2014(24): 51-52

[5] Chen Zixin, Su Xuehen, Liu Shaozong. Research on Ecological Benefits of Urban Garden Greening in Beijing [J], Chinese Landscape Architecture, 2015(1): 57-60

[6] Wen He, Zhou Jiwei. Current Situation and Problems in Modern Urban Plant Landscape Design [J], Urban Construction Theory Research, 2014(12): 33-36

[7] Wang Yibing. Perfect Unity of Nature and Culture-Discussion on Modern Urban Landscape Design [J], Journal of Bohai University, 2015(27): 4-48

[8] Meng Zhaozhen. Gardens in Human Settlement [J], Housing Science, 2015(1): 3-8 